\title{
BMJ Open Effects of moxibustion on reproduction and metabolism of polycystic ovary syndrome: a protocol for meta-analysis and systematic review
}

\author{
Kou Xu (D) , ${ }^{1}$ Jiajie Wang, ${ }^{2}$ Feng Hu, ${ }^{2}$ Siying Lv, ${ }^{1}$ Yanji Zhang, ${ }^{1}$ Qiqi Yang, ${ }^{1}$ \\ Wei Huang, ${ }^{2}$ Zhongyu Zhou ${ }^{2,3}$
}

To cite: Xu K, Wang J, Hu F, et al. Effects of moxibustion on reproduction and metabolism of polycystic ovary syndrome: a protocol for meta-analysis and systematic review. BMJ Open 2021;11:e049039. doi:10.1136/ bmjopen-2021-049039

- Prepublication history and additional supplemental material for this paper are available online. To view these files, please visit the journal online. (http://dx.doi.org/10.1136/ bmjopen-2021-049039).

Received 14 January 2021 Accepted 05 August 2021

\section{Check for updates}

\section{Author(s) (or their} employer(s)) 2021. Re-use permitted under CC BY-NC. No commercial re-use. See rights and permissions. Published by BMJ.

${ }^{1}$ College of Acupuncture and Orthopedics, Hubei University of Chinese Medicine, Wuhan, China ${ }^{2}$ Department of Acupuncture, Hubei Provincial Hospital of Traditional Chinese Medicine, Wuhan, China

${ }^{3}$ Project Team of Acupuncture and Moxibustion Treatment of Dominant Diseases, Evidencebased Medicine Center of Traditional Chinese Medicine in China, Wuhan, China

Correspondence to Professor Zhongyu Zhou; 2209447940@qq.com

\section{ABSTRACT}

Introduction Polycystic ovary syndrome (PCOS) is one of the most common endocrinopathy in women of reproductive age. Recently, moxibustion, as a complementary and alternative therapy, has been commonly used in assisted reproduction and improvement of metabolic abnormalities in patients with PCOS. Currently, intervention efficacy of the use of moxibustion in PCOS treatment still remains controversial due to lack of high-quality evidence. Consequently, this study protocol was designed to objectively review and evaluate the effectiveness and safety of moxibustion treatment for PCOS.

Methods and analysis Electronic searches will be carried out from inception to May 2021 in the online databases of The Cochrane Library, PubMed, EMBASE, Chinese Biomedical Literature, Chongqing VIP Chinese Science and Technology Periodical Database (VIP) and China National Knowledge Infrastructure. The Chinese Clinical Trial Registry Center and Clinical Trials will be used for searching ongoing trials. Randomised controlled trials and the first period in randomised cross-over trials involving any type of moxibustion for patients with PCOS will be included. Primary outcomes will be the ovulation rate, pregnancy rate and sex hormone levels, and secondary outcomes will be changes in clinical symptoms and metabolic indicators, total effective rate and the incidences of side effects and adverse events. Briefly, two reviewers will independently conduct study selection and data extraction, and the risk of bias will be assessed. Prior to the formal meta-analysis, the heterogeneity of included studies will be assessed. Review Manager Statistical Software (RevMan) V.5.3 will be used for data processing. Finally, the Grading of Recommendations Assessment, Development and Evaluation method will be applied to evaluate the quality of evidence.

Ethics and dissemination Ethical approval is not necessary since this study is designed as a systematic review. This study will be disseminated by a peer-review journal or conference presentation.

\section{INTRODUCTION}

Polycystic ovary syndrome (PCOS) is one of the most common endocrine diseases with a $4 \%-21 \%$ prevalence in women of
Strengths and limitations of this study

- This review will be the first systematic review and meta-analysis aiming to evaluate the effects of moxibustion on reproduction and metabolism of polycystic ovary syndrome (PCOS).

- This protocol will be reported in compliance with the Preferred Reporting Items for Systematic Review and Meta-Analysis Protocols guidelines.

- The quality of evidence will be assessed according to the Grades of Recommendations Assessment Development and Evaluation guidelines.

- In order to avoid reviewer bias as much as possible, study screening, data extraction and quality assessment will be carried out by two reviewers separately.

- The main limitation of our study protocol is that some of the included trials may not be of high quality, which will affects our assessment of the risks and benefits of moxibustion in the treatment of PCOS.

reproductive age. PCOS is characterised by oligomenorrhoea or amenorrhoea, infertility, hyperandrogen signs and polycystic ovarian morphology with or without metabolic abnormalities like obesity, insulin resistance and dyslipidaemia. ${ }^{1-3}$

To our best knowledge, PCOS poses a huge impact on fertility. ${ }^{134} \mathrm{~A}$ total of $70 \%-90 \%$ of ovulation disorders and $75 \%$ of anovulatory infertility can be attributed to PCOS. Meanwhile, PCOS also has a negative impact on pregnancy outcomes. ${ }^{35}$ Patients with PCOS also suffer significantly increased risks of metabolic disorders, such as type 2 diabetes mellitus (T2DM), hypertension, obesity, dyslipidaemia and cardiovascular diseases. ${ }^{134}$ For example, the prevalence of dyslipidaemia in patients with PCOS is about $70 \%$, and that of obesity ranges $30 \%-60 \%$. Seriously, the prevalence of impaired glucose tolerance (IGT) in patients with PCOS is up to $31 \%-35 \%$, which is about 20 times higher than that of healthy women of the same age. 
Metabolic disorders increase the risk of cardiovascular and cerebrovascular diseases in patients with PCOS, and aggravate the symptoms of PCOS, leading to a unsatisfactory prognosis. ${ }^{3-6}$

At present, effective cure for PCOS is scant due to its unclear aetiology. Symptomatic treatment has been the main treatment for a long time. ${ }^{7}$ Recommended by the clinical guideline, clomiphene citrate and metformin are preferred as the first-line treatment in patients with PCOS with infertility or severe metabolic disorders such as T2DM and IGT. Hormonal contraceptives (HCs) are also the first-line management for menstrual abnormalities and hirsutism/acne of PCOS. ${ }^{6-8}$ However, in clinical practice, pharmacological interventions can be restricted by multiple factors. For example, HCs are not suitable for patients with PCOS with fertility needs. ${ }^{9}{ }^{10}$ Metformin is not recommended to be taken by patients with heart, liver and kidney dysfunction. ${ }^{7}$ In addition, long-term medication might cause many potential inconveniences and adverse events to the patients. ${ }^{11-15}$ Serving as a type of non-pharmacological interventions, lifestyle interventions (ie, exercise, dieting) are mainly suitable for obese or overweight patients with PCOS. In order to promote fertility, invasive therapies, such as laparoscopic ovarian drilling and in vitro fertilisation-embryo transfer are only used following the non-responsiveness of pharmacological interventions, because they are invasive procedures that cause damages to the patient's body. ${ }^{78}$ Therefore, it is necessary and urgent to search for effective and safe alternative therapy for PCOS. Nowadays, moxibustion is commonly used to assist reproduction and regulate metabolic disorders in patients with PCOS. Evidence for its efficacy, however, is limited.

Moxibustion is a non-penetrating therapy involving burning moxa on relevant acupoints to prevent and treat diseases. It has been widely used in China for thousands of years owing to its simplicity, convenience, effectiveness and low price. ${ }^{16-19}$ It is generally considered that moxibustion exerts the therapeutic role mainly through infrared heat stimulation to human body and pharmacological action of the moxa strip itself. Latest researches have confirmed the biological function of moxibustion in regulating the body's nerve-endocrine-immune network, blood circulation system and metabolism in two directions to restore and maintain the body's health. ${ }^{20}$ Previous clinical studies and reviews in China have indicated the efficacy of moxibustion in relieving clinical symptoms of PCOS (eg, irregular menstruation and obesity), alleviating metabolic abnormalities, increasing ovulation and pregnancy rates, and hormone levels, and improving life quality of patients with PCOS..$^{21-33}$ Therefore, we believed that moxibustion has the therapeutic potential in PCOS.

However, the effects of moxibustion for PCOS have not yet been fully confirmed because high credibility evidences are scant. Therefore, we designed this metaanalysis to review and estimate the effects of moxibustion on reproduction and metabolism of PCOS, aiming to provide references for clinical treatment.
METHODS

\section{Study report and design}

This protocol was reported in compliance with the guidelines of Preferred Reporting Items for Systematic Review and Meta-Analysis (PRISMA) Protocols (see online supplemental appendix A) ${ }^{34}$ Inclusion criteria will include the type of studies, baseline characteristics of participants, interventions and therapeutic outcomes. Any change of the review will be described if needed.

\section{Criteria for inclusion of studies in the review \\ Types of studies}

The review will include randomised controlled trials (RCTs) and the first period in randomised cross-over trials involving any type of moxibustion for patients with PCOS without language limitation.

\section{Types of participants}

Women meeting the revised Rotterdam criteria for PCOS will be included, ${ }^{485}$ regardless of age, race or educational and economic status. Patients with pathological conditions that might cause abnormal ovulation and other serious diseases such as cancer, liver disease and kidney disease will be excluded.

\section{Types of interventions}

Interventions in the treatment group will include any kind of moxibustion, such as needle warming moxibustion, thermal moxibustion, Du moxibustion, thunder-fire moxibustion, indirect moxibustion, mild moxibustion, etc. Moxibustion combined with other conservative treatments (eg, acupuncture, oral medication) will also be included. Differences in the material and origin of moxa sticks and the frequency and time of moxibustion will not be considered.

The controls can be placebo, sham moxibustion, pharmacotherapy, blank control or lifestyle management such as diet and exercise. However, any single or combination therapy of moxibustion will not be considered.

Types of outcome measures

\section{Primary outcomes}

1. Ovulation rate.

2. Pregnancy rate.

3. Sex hormone levels of luteinising hormone (LH), follicle stimulating hormone (FSH), LH/FSH ratio and testosterone.

\section{Secondary outcomes}

1. Total effective rate (TER).

2. Clinical outcomes: Monthly menstrual frequency, Ferriman-Gallway score, body mass index and waist to hip ratio.

3. Glucose and lipid metabolism outcomes: Fasting insulin, fasting blood glucose, total cholesterol, triglycerides, high-density lipoprotein cholesterol and low-density lipoprotein cholesterol.

4. Side effects and adverse events. 


\section{Exclusion criteria}

1. Non-RCTs reviews, case reports, observational studies, animal experimental studies, expert experience and conference articles will be excluded.

2. Duplicate publication.

\section{Search methods for study identification \\ Electronic searches}

Electronic searches will be carried out up from inception to May 2021 in the databases of The Cochrane Library, PubMed, EMBASE, Chinese Biomedical Literature, Chongqing VIP Chinese Science and Technology Periodical Database (VIP) and China National Knowledge Infrastructure and on the registers of The Chinese Clinical Trial Registry Center and Clinical Trials. The combination of Medical Subject Headings and key word terms will be used without language limitation to cross search at all databases and websites, thus avoiding literature missing. The full search strategy for PubMed was shown in online supplemental appendix B, including all search terms. The same strategy will be applied to other databases and registers after appropriate translation.

\section{Searching other resources}

The previous relevant reviews conducted on moxibustion for PCOS and reference lists of included studies will also be searched for more relevant articles. At last, the search will be performed again prior to the final meta-analysis, thus ensuring the eligible literature is all included.

\section{Data collection and analysis \\ Selection of studies}

All reviewers will receive professional training to understand the objective and process of the review before study selection. All the studies retrieved from the electronic database will be imported into the NoteExpress V.3.2.0 software for classification management. First of all, duplicate studies will be excluded. Second, two reviewers ( $Q Y$ and SL) will independently read the titles and abstracts of the studies to screen out eligible ones. Then, two reviewers ( $Q Y$ and $Y Z$ ) will read the full text to determine whether they are eligible for inclusion. Any disagreement will be dissolved by the third reviewer (SL). The procedure selected for the study will be executed according to the PRISMA flow chart (figure 1).

\section{Data extraction and management}

Two independent reviewers (QY and YZ) will extract the following information from included studies:

1. Study characteristics: Country, first author, title, journal, publication year, method of randomisation and blinding method.

2. Participants characteristics: Inclusion and exclusion criteria, sample size, age, duration of disease, symptoms and signs for PCOS.

3. Interventions and comparators: Type, dosage, frequency and course of interventions and comparators, acupoint selection of moxibustion.
4. Outcomes: Primary and secondary outcomes, duration of follow-up and adverse events.

Any disagreement noticed in the process of data crosschecking will be discussed with the third reviewer (KX).

\section{Assessment of risk of bias in included studies}

Two reviewers (QY and YZ) will assess the methodological quality of all RCTs based on the Cochrane Risk of Bias Assessment tool of Cochrane Reviewer's Handbook, including seven aspects: (1) Random sequence generation, (2) allocation concealment, (3) blinding of participants and personnel, (4) blinding of outcome data, (5) incomplete outcome data, (6) selective reporting and (7) other bias. ${ }^{36}$ The studies will be evaluated as being of 'low risk of bias,' 'high risk of bias,' or 'unclear risk of bias'. Any disagreement will be resolved by consensus or arbitrated by the third rater $(\mathrm{KX})$.

\section{Measures of treatment effect}

Dichotomous variables will be summarised using risk ratio value with $95 \%$ CIs. Continuous variables will be summarised using the mean difference/standardised mean difference with $95 \%$ CIs.

\section{Unit of analysis issues}

Before statistical analysis, the units of the outcomes from different studies will be unified according to the International System of Units.

\section{Dealing with missing data}

Relevant authors will be contacted via email or telephone for supplementing incomplete or ambiguous data if necessary. The analysis will be performed based on the current available data if the reply is not obtained.

\section{Assessment of heterogeneity}

Before the formal meta-analysis, $\chi^{2}$ test and $\mathrm{I}^{2}$ statistic will be obtained by RevMan V.5.3 to assess the heterogeneity of included studies qualitatively and quantitatively. $\mathrm{p}<0.1$ of the $\chi^{2}$ test and $\mathrm{I}^{2}>50 \%$ indicate statistically significant heterogeneity between the included studies. ${ }^{36}$

\section{Assessment of reporting biases}

When the number of eligible RCTs $\geq 10$, the reporting bias will be evaluated by generating a funnel plot and conducting Egger's test. ${ }^{37} 38$

\section{Data synthesis}

Data will be synthesised and analysed by RevMan V.5.3. According to the Cochrane Handbook, a fixed effect model will be used to calculate the relative risk and weighted mean difference if the heterogeneity is not statistically significant ( $\mathrm{p} \geq 0.10$ and $\left.\mathrm{I}^{2} \leq 50 \%\right)$; otherwise, a random-effect model will be used to synthesise the data. If the heterogeneity is too great to make the meta-analysis feasible, the results of this study will be qualitatively summarised. 


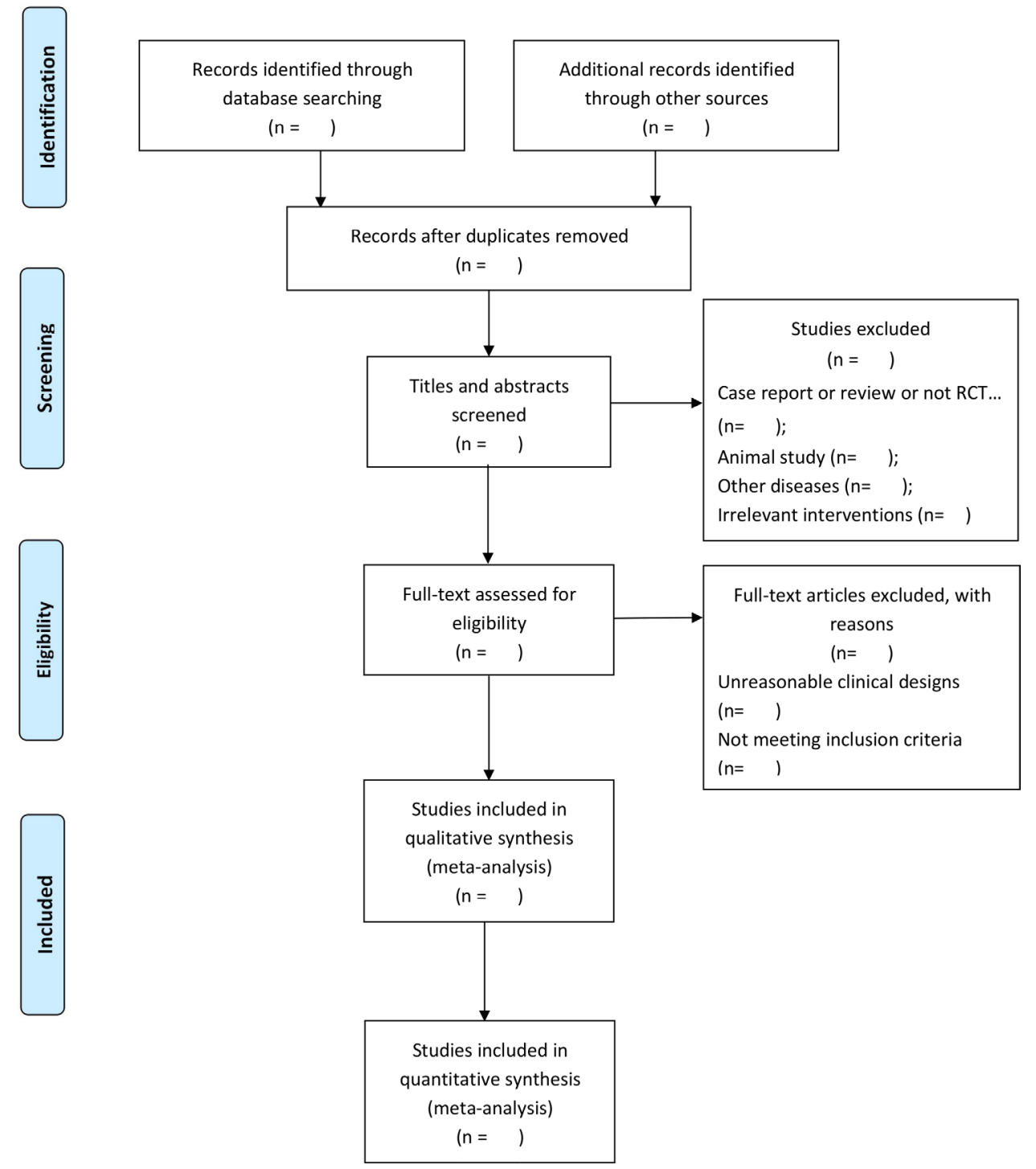

Figure 1 Flow diagram of the study selection process. RCT, randomised controlled trial.

\section{Subgroup analysis}

Subgroup analysis will be performed to probe possible sources of heterogeneity if each subgroup has adequate studies and available data. Criteria of subgroup analysis were as follows:

1. Different types of moxibustion: Direct moxibustion, indirect moxibustion, warm needling moxibustion, thunder-fire moxibustion, Du moxibustion, heat sensitive moxibustion, etc.

2. Different controls: Conventional western medicine treatment or no treatment.

\section{Sensitivity analysis}

If heterogeneity remains between included studies after subgroup analysis, sensitivity analysis will be performed to reperform this meta-analysis after excluding studies with high-risk bias (eg, small sample size, methodological weaknesses and missing data), thus ensuring the robustness of analysis results. The results of two successive metaanalysis will be carefully compared and discussed to yield the final conclusion.
Evidence quality evaluation

Grades of Recommendations Assessment Development and Evaluation (GRADE) profiler V.3.6 software will be used to evaluate the quality of evidence according to the GRADE guidelines. ${ }^{39}$ The specific evaluation method was as follows: The quality improved based on the three factors of residual confounding, dose-response gradient and large magnitude of effect, and the quality was reduced by the five factors of study limitation, inconsistency, indirectness, publication bias and imprecision. Qualities of included studies will be categorised into very low, low, moderate and high.

\section{Patient and public involvement}

This study protocol did not involve either patients or the public.

\section{Amendments}

If there are any amendments to the protocol, we will explain in the final report. 


\section{Ethics and dissemination}

Ethical approval is not necessary since this protocol is only for systematic review that does not involve privacy data or conduct an animal experiment. This protocol was disseminated by a peer-review journal or conference presentation.

Contributors KX, JW and FH contributed equally to this work. All authors have read and approved the publication of the protocol. Conceptualisation: KX, ZZ and WH. Data curation: QY, SL and YZ. Formal analysis: WH, KX and QY. Investigation: $K X$ and JW. Methodology: WH, FH, KX and YZ. Software: JW, QY and SL. Supervision: FH, WH and ZZ. Writing-original draft: KX, QY, SL and JW. Writing-review and editing: JW, ZZ, WH and $\mathrm{FH}$.

Funding This work was supported by: 2020 National Administration of Traditional Chinese Medicine 'Special Project for the Inheritance of Ancient Chinese Medicine Documents and Characteristic Technologies', grant number: Guozhong Medical Science and Technology Letter (2020) No. 295; Hubei Provincial Health Commission 2021-2022 Traditional Chinese Medicine Research Project, grant number: E Weitong (2020) No. 38; The 2nd Hubei Province's Outstanding Medical Academic Leader Program, grant number: No. (2019)47; National Administration of Traditional Chinese Medicine: 2019 Project of building evidence based practice capacity for TCM, grant number: No.2019XZZX-ZJ006; Qihuang Engineering Project of National Administration of Traditional Chinese Medicine, grant number: No. 284 (2018); 2020 Project in the Co-innovation Center for Preventive Treatment of Disease of Acupuncture-moxibustion in Hubei Province, grant number: HBPCIC-2020-05, HBPCIC-2020-11; Hubei hospital of traditional Chinese medicine, the first Tanhualin famous doctor, student training project, grant number: (2018) no. 72; The Project of Hubei Famous Doctor of Traditional Chinese Medicine Studio, grant number: No. 15 (2018); 2015 Special Project in TCM Industry of State of Administration of Traditional Chinese Medicine of the People's Republic of China, grant number: 201507003; National Natural Science Foundation of China, grant number: 81674081; Wuhan young and middle-aged medical backbone talents (sixth batch), grant number: no. 116, family planning tong (2018).

Competing interests None declared.

Patient consent for publication Not required.

Provenance and peer review Not commissioned; externally peer reviewed.

Supplemental material This content has been supplied by the author(s). It has not been vetted by BMJ Publishing Group Limited (BMJ) and may not have been peer-reviewed. Any opinions or recommendations discussed are solely those of the author(s) and are not endorsed by BMJ. BMJ disclaims all liability and responsibility arising from any reliance placed on the content. Where the content includes any translated material, BMJ does not warrant the accuracy and reliability of the translations (including but not limited to local regulations, clinical guidelines, terminology, drug names and drug dosages), and is not responsible for any error and/or omissions arising from translation and adaptation or otherwise.

Open access This is an open access article distributed in accordance with the Creative Commons Attribution Non Commercial (CC BY-NC 4.0) license, which permits others to distribute, remix, adapt, build upon this work non-commercially, and license their derivative works on different terms, provided the original work is properly cited, appropriate credit is given, any changes made indicated, and the use is non-commercial. See: http://creativecommons.org/licenses/by-nc/4.0/.

ORCID iD

Kou Xu http://orcid.org/0000-0003-0200-0576

\section{REFERENCES}

1 Azziz R, Carmina E, Chen Z, et al. Polycystic ovary syndrome. Nat Rev Dis Primers 2016;2:16057.

2 Lizneva D, Suturina L, Walker W, et al. Criteria, prevalence, and phenotypes of polycystic ovary syndrome. Fertil Steril 2016;106:6-15.

3 Liu W. Polycystic ovary syndrome and endocrine infertility. Shanghai: Shanghai Science and Technology Press, 2016: 24-52.

4 Legro RS, Arslanian SA, Ehrmann DA, et al. Diagnosis and treatment of polycystic ovary syndrome: an endocrine Society clinical practice guideline. J Clin Endocrinol Metab 2013;98:4565-92.
5 Li R, Zhang Q, Yang D, et al. Prevalence of polycystic ovary syndrome in women in China: a large community-based study. Hum Reprod 2013;28:2562-9.

6 Chen X, Yang D, Li L, et al. Abnormal glucose tolerance in Chinese women with polycystic ovary syndrome. Hum Reprod 2006;21:2027-32.

7 Endocrinology Subgroup and Expert Panel, Chinese Society of Obstetrics and Gyneocology, Chinese Medical Association. [Chinese guideline for diagnosis and management of polycystic ovary syndrome]. Zhonghua Fu Chan Ke Za Zhi 2018;53:2-6.

8 Teede HJ, Misso ML, Costello MF, et al. Recommendations from the International evidence-based guideline for the assessment and management of polycystic ovary syndrome. Clin Endocrinol 2018;89:251-68.

9 Kwon C-Y, Lee B, Park KS. Oriental herbal medicine and moxibustion for polycystic ovary syndrome: a meta-analysis. Medicine 2018;97:e12942.

10 Rocca ML, Venturella R, Mocciaro R, et al. Polycystic ovary syndrome: chemical pharmacotherapy. Expert Opin Pharmacother 2015;16:1369-93.

11 Liu M, Zhu H, Hu X, et al. Efficacy of different forms of Guizhi Fuling Wan on reproduction and metabolism in women with polycystic ovary syndrome: a protocol for a meta-analysis of randomized controlled trials. Medicine 2020;99:e22954.

12 Diamanti-Kandarakis E, Baillargeon J-P, luorno MJ, et al. A modern medical quandary: polycystic ovary syndrome, insulin resistance, and oral contraceptive pills. J Clin Endocrinol Metab 2003;88:1927-32.

13 Legro RS, Barnhart HX, Schlaff WD, et al. Clomiphene, metformin, or both for infertility in the polycystic ovary syndrome. N Engl J Med 2007;356:551-66.

14 Costello M, Shrestha B, Eden J, et al. Insulin-Sensitising drugs versus the combined oral contraceptive pill for hirsutism, acne and risk of diabetes, cardiovascular disease, and endometrial cancer in polycystic ovary syndrome. Cochrane Database Syst Rev 2007;1:CD005552.

15 Nasri H, Rafieian-Kopaei M. Metformin: current knowledge. J Res Med Sci 2014;19:658-64.

16 Liu XS, Deng LL. Practical Handbook of moxibustion. Beijing: China Press of Traditional Chinese Medicine, 2017.

17 Zhang T, Wang L-P, Wang G-L, et al. Effects of moxibustion on symptoms of mild cognitive impairment: protocol of a systematic review and meta-analysis. BMJ Open 2020;10:e033910.

18 Leem J, Lee S, Park Y, et al. Effectiveness and safety of moxibustion treatment for non-specific lower back pain: protocol for a systematic review. BMJ Open 2017;7:e014936.

19 Chen S, Guo S, Wang J, et al. Effectiveness of moxibustion for allergic rhinitis: protocol for a systematic review. BMJ Open 2015;5:e006570.

20 Wang L, XW L, Zhang L. Advances of studies on mechanisms of moxibustion therapy at home and abroad. Zhongguo Zhen Jiu 2001;09:56-9.

21 Yang J, Lin Q-P, You X-M, et al. [Effect of combined treatment with acupuncture, moxibustion and medication on endometrial receptivity and expression of serum HOXA10 in polycystic ovary syndrome of kidney deficiency and blood stagnation]. Zhongguo Zhen Jiu 2020;40:1154-8.

$22 \mathrm{HXX}$, Zhu CL, Tang H. Effect of moxibustion combined with Chinese medication on ovarian reserve function in patients with infertility due to polycystic ovary syndrome. Shanghai J Acu-mox 2021;40:571-5.

23 LL Y, Guo L, Shang S. Effect of Yijingyangshenyunzi decoction combined with moxibustion on sex hormone level and ovarian hemodynamics in infertile women with polycystic ovary syndrome (Chinese). Hubei journal of TCM 2020;42:13-15.

24 Xie J. Moxibustion combined with traditional Chinese medicine in the treatment of polycystic ovary syndrome with phlegm dampness syndrome:A Randomized Placebo Control Trial. Chengdu University of TCM, 2017.

25 Chen MR. The efficacy observation of treating the phlegm stagnation type polycystic ovarian syndrome by moxibustion combined with sequentially taking the Chinese medicine. Guangzhou University of TCM, 2015 .

26 Zhang GP. Clinical study on treatment of hyperandrogenism in polycystic ovary syndrome with abdominal acupuncture and moxibustion. Guangzhou University of TCM, 2012.

$27 \mathrm{XQ}$ L, Shi XL. Clinical observation of moxibustion for ovulation disorders (Chinese). Zhongguo Zhen Jiu 1999;12:23-4.

28 Yang J, Liu Y, Huang J, et al. [Acupuncture and Chinese medicine of artificial cycle therapy for insulin resistance of polycystic ovary syndrome with phlegm damp type and its mechanism]. Zhongguo Zhen Jiu 2017;37:1163-8. 
29 Song Y-J, Liang F-X, Wu S, et al. [Network meta-analysis on the effects of the acupuncture-related therapy on ovulation rate and pregnancy rate in patients with polycystic ovary syndrome]. Zhongguo Zhen Jiu 2019;39:792-8.

30 TJ Y, Cheng HX. Therapeutic efficacy of moxibustion plus medicine in the treatment of infertility due to polycystic ovary syndrome and its effect on serum immune inflammatory factors. J Acupunct Tuina 2020;18:269-75.

31 Hong L, Licheng F. Study on effects of acupuncture on menstruation and endocrine in patients of normal body weight with polycystic ovary syndrome. World Journal of Acupuncture-Moxibustion 2014;24:10-14.

32 Chen YR. Clinical study of moxibustion on the Zigong point in polycystic ovary syndrome induced ovulation(Chinese). China Practical Medicine 2017;12:117-8.

33 YG X. Curative effect observation of 128 cases of polycystic ovary syndrome infertility with spleen and kidney yang deficiency type treated with traditional Chinese medicine and moxibustion(Chinese). World Chinese Medicine 2014;09:1079-82.
34 Moher D, Shamseer L, Clarke M, et al. Preferred reporting items for systematic review and meta-analysis protocols (PRISMA-P) 2015 statement. Syst Rev 2015;4:1.

35 Rotterdam ESHRE/ASRM-Sponsored PCOS Consensus Workshop Group. Revised 2003 consensus on diagnostic criteria and longterm health risks related to polycystic ovary syndrome. Fertil Steril 2004;81:19-25

36 Green S, Higgins JPT, The Cochrane Collaboration. Cochrane Handbook for systematic reviews of interventions. version 5.1.0. The Cochrane collaboration, 2011.

37 Sutton AJ, Duval SJ, Tweedie RL, et al. Empirical assessment of effect of publication bias on meta-analyses. BMJ 2000;320:1574-7.

38 Egger M, Davey Smith G, Schneider M, et al. Bias in meta-analysis detected by a simple, graphical test. BMJ 1997;315:629-34.

39 Guyatt G, Oxman AD, AkI EA, et al. Grade guidelines: 1. IntroductionGRADE evidence profiles and summary of findings tables. J Clin Epidemiol 2011;64:383-94. 